\title{
Programming plasma cell survival
}

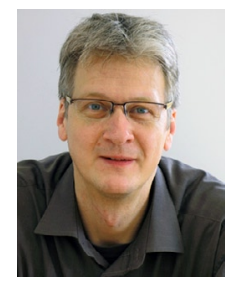

Insight from Ulf Klein

In this issue, two independent groups show that the transcription factor ZBTB20 is required for the maintenance of long-lived plasma cells following immunization with the commonly used adjuvant, alum. Intriguingly, one group found that ZBTB20 was dispensable for plasma cell survival when they used adjuvants that activate Toll-like receptors (TLRs).

Protection from recurrent infections depends on long-lived plasma cells secreting high-affinity antibodies, which are generated during $\mathrm{T}$ cell-dependent immune responses in the germinal center reaction. But not all vaccinations or infections yield durable and protective plasma cell responses. Therefore, a better understanding of the molecular mechanisms that govern plasma cell generation and maintenance is needed in order to develop more effective vaccines.

Chevrier et al. and Wang and Bhattacharya now show that the BTB-POZ transcription factor ZBTB20, which is expressed in germinal center B cells and at high levels in plasma cells, is crucial for maintaining durable antibody responses by promoting cell survival following immunization of mice with a model antigen in alum. But when Wang and Bhattacharya administered the same antigen with TLR-ligand based adjuvants they observed that ZBTB20 was dispensable for the development and survival of plasma cells. Both ZBTB20-dependent and -independent plasma cell survival pathways were B cell-intrinsic, suggesting that the immune cells activated by the corresponding adjuvants imprint longevity on the plasma cells when they are formed within the germinal centers.

The mechanisms underlying this imprinting process are presently unknown and require further investigation. Chevrier et al. show clearly that the control of ZBTB20 expression differs fundamentally between germinal center B cells and plasma cells. $Z b t b 20$ is a transcriptional target of the plasma cell master regulator IRF4 in plasma cells, but not in the majority of germinal center B cells, which lack IRF4. However, IRF4 expression is up-regulated in a small subset of late germinal center B cells that are plasma cell precursors. It is therefore possible that the ZBTB20-dependent survival program is initiated at this developmental stage through interaction with $\mathrm{T}$ follicular helper cells. Similarly, this particular germinal center subpopulation may be the downstream target of TLR-ligand based adjuvants. It will be important to identify the ZBTB20-equivalent transcription factor, which Wang and Bhattacharya suggest might be another BTB-POZ transcription factor, ZBTB32.

An important implication of the current studies is that we need to dissect the transcription factor network that controls plasma cell generation within the germinal center in order to understand how adjuvants improve the durability of vaccines. In addition, the results clearly indicate that

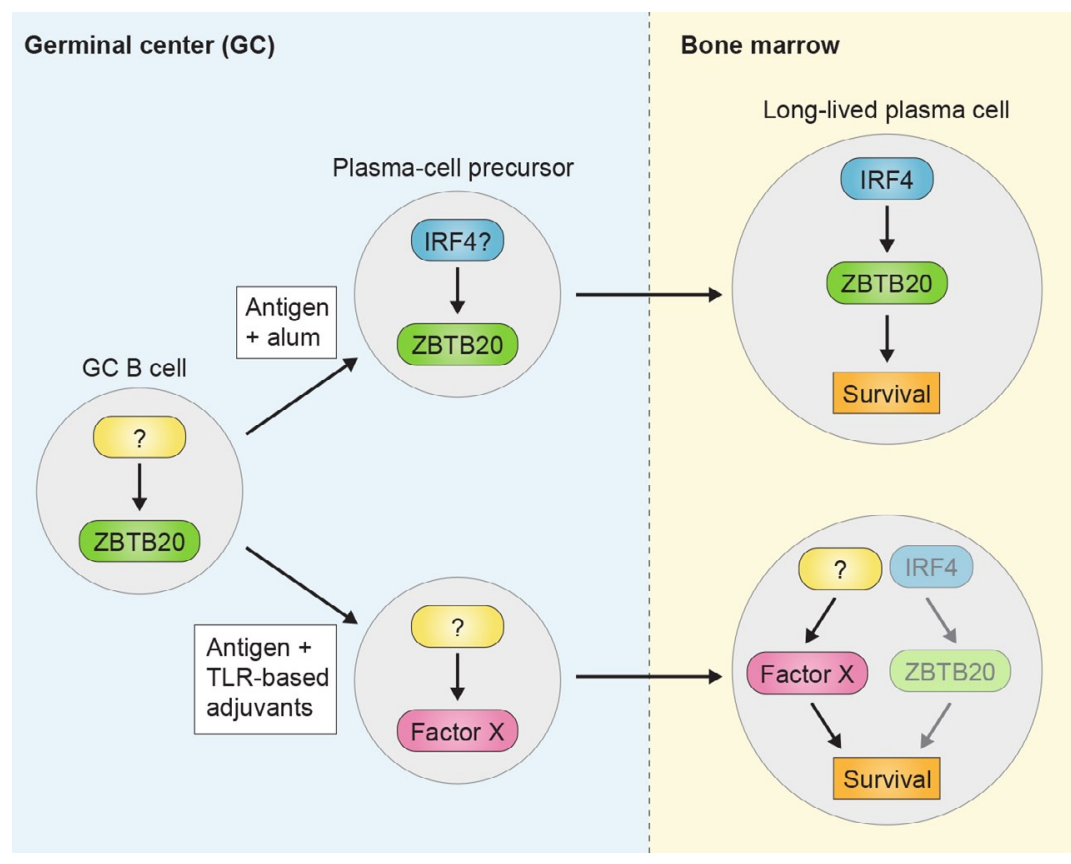

Model of how different adjuvants may imprint distinct plasma cell survival programs in late germinal center B cells. in vaccine development, it is important to know whether an infection triggers an alum-like or TLR-biased response. The findings also serve as a reminder to be more cautious with generalizing results obtained from the study of a single immunization model.

Chevrier, S., et al. 2014.J. Exp. Med. http://dx.doi.org/10.1084/jem.20131831.

Wang,Y., and D. Bhattacharya. 2014.J. Exp. Med. http://dx.doi.org/10.1084/jem.20131821.

Ulf Klein, Columbia University: uk30@columbia.edu 


\section{Lymphatics help baby take first breath}

Using mutant mice that lack lymph vessels, Jakus et al. show that prenatal lymphatic function is required for neonatal lung inflation and respiration.

In the womb, a baby's lungs are filled with fluid that must be cleared close to birth to allow neonatal lung inflation and respiration. The bulk of the fluid is cleared by active transepithelial absorption from the airways into the lung interstitial spaces, a process that starts before birth in response to hormonal changes.

Jakus et al. observed that in mice that lack the gene encoding CCBE1 or that express a dysfunctional VEGFR3 receptor, molecules involved in lymph vessel formation, the majority of the newborn pups are bluish in color and do not start to breathe. Although the lungs develop and mature normally, they fail to inflate and the mice die shortly after birth.

In healthy wild-type late-gestation embryos, the authors found that pulmonary lymphatics are already functioning. But in the mutant embryos, the absence of lymphatic function was associated with accumulation of fluid in the lung tissue and with reduced ability of the prenatal lung to expand (a feature known as lung compliance). Based on these observations, Jakus et al. propose a model in which pulmonary lymphatic function

\section{Wild-type embryo}

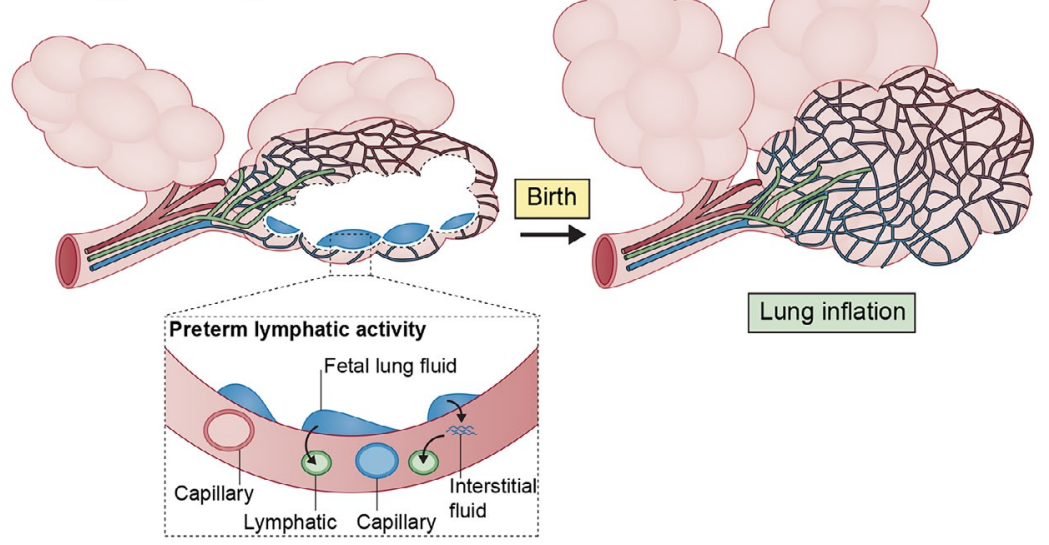

Embryo lacking lymphatics

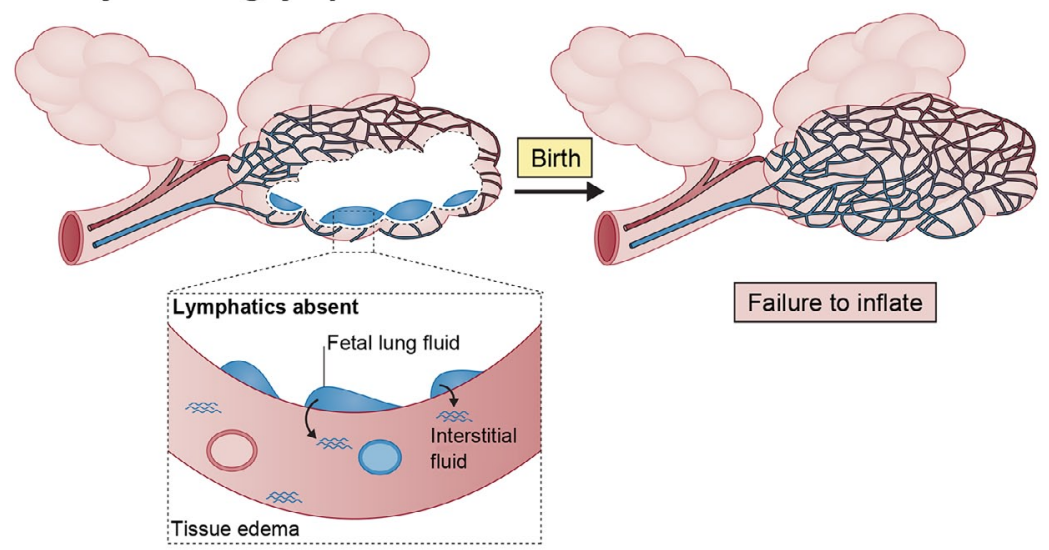

Lungs of prenatal mice lacking lymphatics display impaired drainage of interstitially absorbed lung fluid during preparation for birth; the newborn mice then fail to inflate their lungs and can't breathe.

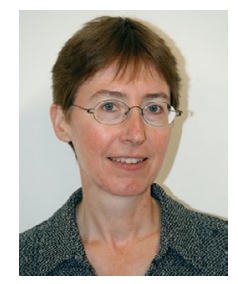

Insight from Mieke Dewerchin in preterm mice is required for drainage of absorbed fluid in the lungs, thus preventing tissue edema and securing intrinsic lung compliance prior to birth. In this way, prenatal lymphatic function helps prepare the lungs for inflation and gas exchange after birth.

It was reported previously that lymphatics contribute to the clearance of remaining lung fluid in newborns and that lymphatic hypoplasia compromises neonatal survival. Jakus et al. extend these findings by revealing the importance of functional pulmonary lymphatics prior to birth. Besides other crucial mechanisms, such as surfactant production, prenatal lymphatic function appears to be an additional mechanism required to prepare the lung for inflation, which is a previously unrecognized role of the lymphatic system.

The authors observed differences in phenotype severity depending on the genetic background of the mutant mice, suggesting the influence of as yet unidentified modifying factors. Nevertheless, further investigation into temporal aspects of prenatal lymphatic drainage may elucidate whether inadequate prenatal lymphatic function contributes to respiratory distress syndrome in premature infants, and may explain respiratory failure in infants with congenital pulmonary lymphangiectasia, a rare disorder of lung lymphatic development.

Jakus, Z., et al. 2014. J. Exp. Med. http://dx.doi .org/10.1084/jem.20132308.

Mieke Dewerchin, VIB Vesalius Research Center, KU Leuven: mieke.dewerchin@vib-kuleuven.be 


\section{$\mathrm{B}-\mathrm{RAF}$ unlocks axon regeneration}

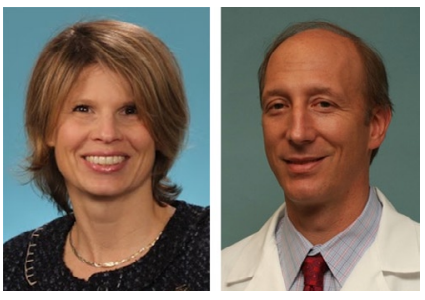

Insight from Valeria Cavalli (left) and David Holtzman
The mechanisms that drive axon regeneration after central nervous system (CNS) injury or disease are proposed to recapitulate, at least in part, the developmental axon growth pathways. This hypothesis is bolstered by a new study by O'Donovan et al. showing that activation of a B-RAF kinase signaling pathway is sufficient to promote robust axon growth not only during development but also after injury.

B-RAF was previously shown to be essential for developmental axon growth but it was not known if additional signaling pathways are required. In this study, the authors demonstrate that activation of B-RAF alone is sufficient to promote sensory axon growth during development. Using a conditional B-RAF gain-of-function mouse model, the authors elegantly prove that B-RAF has a cell-autonomous role in the developmental axon growth program. Notably, activated B-RAF promoted overgrowth of embryonic sensory axons projecting centrally in the spinal cord, suggesting that this pathway may normally be quiescent in central axons.

Could activated B-RAF also enhance axon regeneration in the adult central nervous system? The authors found that activated B-RAF not only enabled sensory axon growth into the spinal cord after spinal injury, but also promoted regrowth of axons projecting in the optic nerve. Regeneration in the injured CNS is prevented by both the poor intrinsic regrowth capacity of axons and by inhibitory factors in the tissue environment. Importantly, the B-RAF-activated signaling growth program was insensitive to this repulsive environment.

Interestingly, the authors find that B-RAF synergizes with the PI3kinase-mTOR pathway, which also functions downstream of growth factors. This opens the possibility that combinatorial approaches that integrate these two pathways may heighten regenerative capacity.

This in vivo study significantly advances the understanding of the role of MAP kinases in axon growth and suggests that reactivation of the B-RAF pathway may be exploited to promote axon regeneration in the injured central nervous system. An exciting future avenue will be to determine the downstream mechanisms controlled by B-RAF.

O’Donovan, K.J., et al. 2014.J. Exp. Med. http://dx.doi.org/10.1084/jem.20131780.

\section{New role identified for LRR-containing proteins in $\mathrm{T}$ cell development}

There are thousands of leucine-rich repeat (LRR)-containing proteins, which share a common structural horseshoe-shaped motif but have distinct and unrelated functions. Among the LRR-containing proteins involved in the immune system, Toll-like receptors (TLRs) and nucleotide-binding oligomerization domain (NOD)-like receptor proteins (NLRPs) easily come to mind, with important functions in pathogen recognition by innate immune cells. Nevertheless, there are many other LRR-containing proteins whose functions remain to be fully elucidated.

In this issue, Kumar et al. report their work on the role of LRRC8A in lymphocyte development and function. The current study was initiated following identification of a translocation mutation in the LRRcontaining 8A (LRRC8A) gene in a patient with no B cells and agammaglobulinemia, which resulted in a truncated protein that severely inhibited B cell development and function. However, the function of LRRC8A in lymphocytes or its role in other cell lineages is not fully understood.

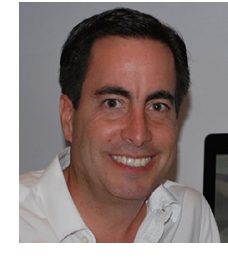

Insight from Juan-Carlos Zúñiga-Pflücker 


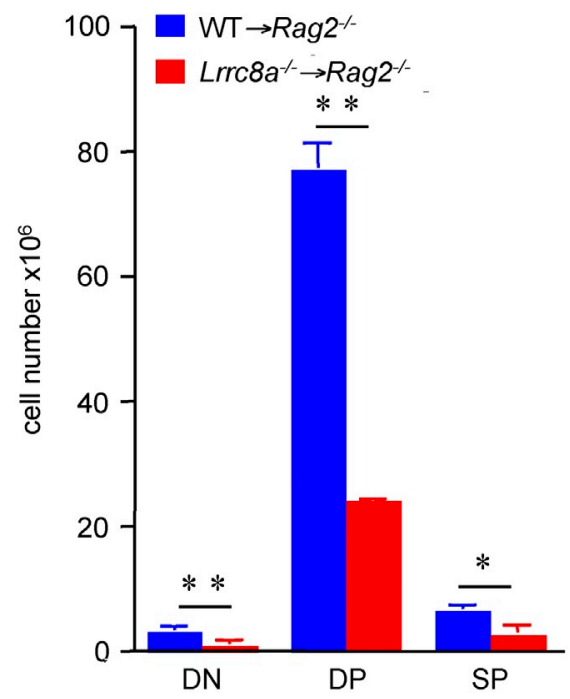

Thymi from $\mathrm{LrrC8}^{-/-}$mice showed a threefold reduction in the numbers of double-negative, double-positive, and single-positive thymocytes compared with wild-type thymi.
The authors generated $\operatorname{Lrc} 8 \mathrm{a}$-deficient mice that showed a defect in both $\mathrm{T}$ cell development and function, with increased thymocyte apoptosis, but only a mild defect in B cell development. LRRC8A, which is expressed on the cell surface, appears to engage a ligand expressed on thymic epithelial cells, as well as on multiple other stromal cells. The authors provide evidence that this interaction leads to AKT activation via an LCK-ZAP-70-GAB2-PI3K signaling pathway in $\mathrm{T}$ cells. However, further work will be required to definitively establish whether the AKT signaling pathway is crucial for the defect in thymocyte development observed in Lrr8a-null mice. In addition to the defect in thymocyte survival and proliferation, $\operatorname{Lrc} 8 a$-deficient mice also exhibited defective $\mathrm{T}$ cell function downstream of the TCR. These findings were unexpected, as the initial characterization of the human LRRC8A mutation had a more severe defect in the development and function of B cells than T cells. This difference may be due to a dominant-negative gain of function of the truncated mutation displaying broader and distinct effects in humans compared with the effects observed in the gene-deficient mice.

These exciting findings point to an as yet unappreciated role for LRRcontaining proteins in $\mathrm{T}$ cell development and function. It is likely that other members of this large gene family may be equally important, and thus the present report serves as a clarion call for further work on LRRC8A-related genes in lymphocyte differentiation and function.

Kumar, L., et al. 2014. J. Exp. Med. http://dx.doi.org/10.1084/jem.20131379. 